\title{
Influence of chromatic dispersion on degenerate four-wave mixing
}

\author{
B. Crosignani \\ Fondazione Ugo Bordoni, Istituto Superiore P.T., Roma, Italy
}

P. Di Porto

Istituto di Fisica dell' Università dell' Aquila, L'Aquila, Italy, and Fondazione Ugo Bordoni, Roma, Italy

Received April 29, 1982

\begin{abstract}
We investigate the way in which four-wave mixing in a waveguide is affected by chromatic dispersion. The frequency dependence of the associated phase-conjugate mirror reflectivity turns out not to be influenced by chromatic dispersion itself.
\end{abstract}

Degenerate four-wave mixing is investigated both in the stationary regime ${ }^{1}$ (in which dispersion has no role) and in the nonstationary regime (in which the time behavior of the phase-conjugate signal is important, as when one is studying the frequency response of a phase-conjugate mirror ${ }^{2}$ ), without taking into account chromatic dispersion. Although this circumstance can be $a$ priori justifiable over the short crystal samples in which four-wave mixing is usually performed, one is conversely led to think that chromatic dispersion can no longer be ignored when the process takes place in long glass fibers.

In this Letter, we study the time-dependent behavior of the phase-conjugate reflected signal by including in the equation describing its evolution the contribution that is due to chromatic dispersion, that is, a term containing $\beta^{\prime \prime}=d^{2} \beta / d \omega^{2}, \beta$ being the propagation constant of the incident signal inside the nonlinear medium $[\beta(\omega)=n(\omega) \omega / c$, where $n(\omega)$ is the material refractive index, if the medium is unbounded]. It turns out that this behavior (or, equivalently, the frequency response of the associated phase-conjugate mirror) is independent of $\beta^{\prime \prime}$, irrespective of the distance traveled in the medium.

Let us write the analytic signals of the electric fields associated with the incident and reflected waves in the form (see Fig. 1)

$$
\begin{aligned}
& \hat{E}_{i}(\mathbf{r}, t)=E(x, y) \exp \left[-i \beta\left(\omega_{0}\right) z+i \omega_{0} t\right] \Phi_{i}(z, t), \\
& \hat{E}_{r}(\mathbf{r}, t)=E(x, y) \exp \left[i \beta\left(\omega_{0}\right) z+i \omega_{0} t\right] \Phi_{r}(z, t),
\end{aligned}
$$

where $\omega_{0}$ is the central angular frequency, $\beta\left(\omega_{0}\right)$ is the associated propagation constant, and $\Phi_{i}$ and $\Phi_{r}$ are slowly varying amplitudes. The system of equations describing degenerate four-wave mixing, in the presence of a nondepleted continuous-wave pump, is ${ }^{3}$

$$
\begin{gathered}
{\left[\partial / \partial z+(1 / V) \partial / \partial t-(i / 2 A) \partial^{2} / \partial t^{2}\right] \Phi_{i}(z, t)} \\
=-i S \Phi_{i}(z, t)-i P \Phi_{r} *(z, t) \\
{\left[\partial / \partial z-(1 / V) \partial / \partial t+(i / 2 A) \partial^{2} / \partial t^{2}\right] \Phi_{r}(z, t)} \\
=i S \Phi_{r}(z, t)+i P \Phi_{i}^{*}(z, t),
\end{gathered}
$$

where

$$
V=(\mathrm{d} \beta / \mathrm{d} \omega)_{\omega=\omega_{0}}^{-1}, \quad A=\left(\mathrm{d}^{2} \beta / \mathrm{d} \omega^{2}\right)_{\omega=\omega_{0}}^{-1}
$$

represent, respectively, the group velocity and the group-velocity dispersion inside the medium, which generalizes the system of equations describing degenerate four-wave mixing in thin media ${ }^{1}$ (where one can put $1 / V=1 / A=0)$ and in relatively thick media ${ }^{4,5}(1 / A$ $=0)$. The coefficients $S$ and $P$ are given $b^{3}$

$$
S=2 \alpha \omega_{0}\left(n_{2} / c\right)\left(\left|\hat{E}_{p}{ }^{(+)}\right|^{2}+\left|\hat{E}_{p}(-)\right|^{2}\right)
$$

and

$$
P=2 \alpha \omega_{0}\left(n_{2} / c\right) \hat{E}_{p}^{(+)} \hat{E}_{p}^{(-)},
$$
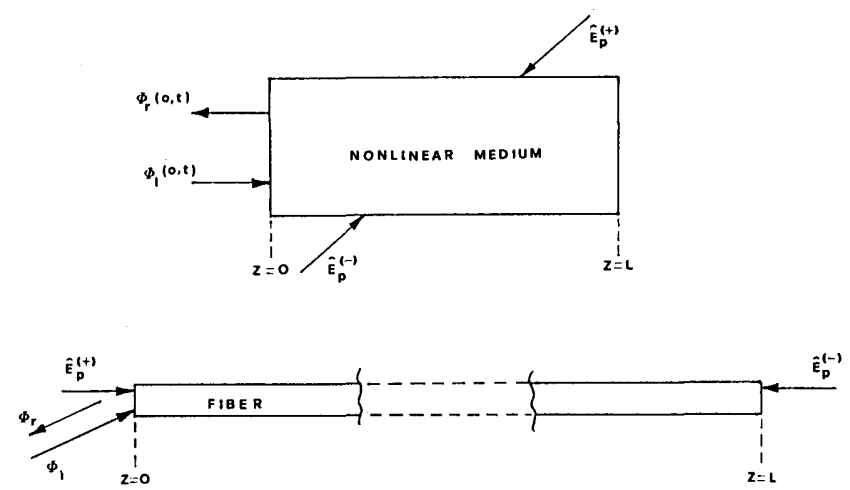

Fig. 1. Four-wave mixing geometry in a short medium and in a long optical fiber. 
where $n_{2}$ is the nonlinear refractive index of the medium and $\alpha$ is a suitable superposition integral between the normalized intensities of the pump and signal modes. If $P$ does not depend on $z$ [which can be shown ${ }^{3}$ to hold true whenever $\left.\left|\hat{E}_{p}{ }^{(+)}(z=0)\right|^{2}=\left|\hat{E}_{p}{ }^{(-)}(z=0)\right|^{2}\right]$, the system of Eqs. (3) can be solved exactly by employing the standard normal-mode expansion, that is, by looking for a solution of the kind

$$
\begin{aligned}
\Phi_{i}(z, t) & =\tilde{\Phi}_{i}(\omega) \exp [i k(\omega) z-i \omega t], \\
\Phi_{r} *(z, t) & =\tilde{\Phi}_{r} *(\omega) \exp [i k(\omega) z-i \omega t] .
\end{aligned}
$$

By proceeding in this way, we obtain

$$
\begin{aligned}
& \Phi_{i}(z, t)=e^{-i S z} \\
& \times \int_{-\infty}^{+\infty} \mathrm{d} \omega G(\omega) \exp \left[-i\left(\omega^{2} z / 2 A\right)-i \omega t\right] \\
& \times \frac{i(\omega / V) \sin [f(\omega)(z-L)]+f(\omega) \cos [f(\omega)(z-L)]}{f(\omega) \cos [f(\omega) L]-(i \omega / V) \sin [f(\omega) L]},
\end{aligned}
$$

$$
\begin{aligned}
& \Phi_{r} *(r, t)=-\left(i|P|^{2} / P^{2}\right) e^{-i S z} \\
& \times \int_{-\infty}^{+\infty} \mathrm{d} \omega G(\omega) \exp \left[-i\left(\omega^{2} z / 2 A\right)-i \omega t\right] \\
& \times \frac{\sin [f(\omega)(z-L)]}{f(\omega) \cos [f(\omega) L]-(i \omega / V) \sin [f(\omega) L]},
\end{aligned}
$$

where $f(\omega)=\left(|P|^{2}+\omega^{2} / V^{2}\right)^{1 / 2}$ and

$$
G(\omega)=(1 / 2 \pi) \int_{-\infty}^{+\infty} \Phi_{i}(z=0, t) e^{i \omega t} \mathrm{~d} t .
$$

In particular, at $z=0$ we have

$$
\Phi_{r} *(z=0, t)=\int_{-\infty}^{+\infty} \mathrm{d} \omega G(\omega) R(\omega) e^{-i \omega t},
$$

having introduced the complex frequency-dependent reflectivity

$$
R(\omega)=-i \frac{|P|^{2}}{P^{2}} \frac{\tan [f(\omega) L]}{(i \omega / V) \tan [f(\omega) L]-f(\omega)} .
$$

Remarkably, the factor $1 / A$ accounting for chromatic dispersion does not appear in Eq. (12). As a matter of fact, Eq. (12) is equivalent to that worked out in Ref. 2, in which the authors consider a dispersionless medium.

Chromatic dispersion affects four-wave mixing only if one takes into account the presence of higher-order group dispersion, which is expressed by terms of the kind

$$
B=\left(\mathrm{d}^{3} \beta / \mathrm{d} \omega^{3}\right)_{\omega=\omega_{0},}^{-1}, \quad C=\left(\mathrm{d}^{4} \beta / \mathrm{d} \omega^{4}\right)_{\omega=\omega_{0}}^{-1}, \ldots
$$

In this case, the system of Eqs. (3) has to be modified by adding on the left-hand side the differential terms $^{3}$

$$
\mp \frac{1}{3 ! B} \frac{\partial}{\partial t^{3}} \pm \frac{i}{4 ! C} \frac{\partial}{\partial t^{4}} \mp \ldots
$$

(the upper and the lower signs applying, respectively, to the first and the second equations). It turns out, however, that only the terms containing odd derivatives with respect to time are capable of affecting the complex reflectivity $R(\omega)$; in other words, if one could neglect all these terms [including $(1 / V) \partial / \partial t$ ], degenerate four-wave mixing would give rise to an exact time-reversed replica of the incident signal.

On physical grounds, the lack of influence of evenorder group dispersion on complex reflectivity can be associated with the property of four-wave mixing of providing a time-reversed replica of the signal. In fact, even-order dispersion corresponds, in the time domain, to terms that are invariant in the exchange $t \rightarrow-t$.

\section{References}

1. See, for example, A. Yariv, "Phase conjugate optics and real-time holography," IEEE J. Quantum Electron. QE-14, 650-660 (1978).

2. D. M. Pepper and R. L. Abrams, "Narrow optical bandpass filter via nearly degenerate four-wave mixing," Opt. Commun. 3, 212-214 (1978); J. AuYeung, D. Fekete, D. M. Pepper, and A. Yariv, "A theoretical and experimental investigation of the modes of optical resonators with phase-conjugate mirrors,' IEEE J. Quantum Electron. QE-15, 1180-1188 (1979).

3. B. Crosignani, "Self-induced nonlinear effects in optical fibers: a unified approach," in New Directions in Guided Waves and Coherent Optics, D. Ostrowsky, ed. (Plenum, New York, to be published).

4. J. H. Marburger, "Optical pulse integration and chirp reversal in degenerate four-wave mixing," Appl. Phys. Lett. 32, 372-374 (1978).

5. R. A. Fisher, B. R. Suydam, and B. J. Feldman, "Transient analysis of Kerr-like phase conjugators using frequencydomain techniques," Phys. Rev. A 23, 3071-3083 (1981). 